\title{
Die vierte Dimension
}

\section{Wissensdiskurse und Imagination in Alan Moores und Eddie Campbells »From Hell«}

Joachim Trinkwitz

Der umfangreiche Comic From Hell der Briten Alan Moore und Eddie Campbell, in unterschiedlicher Form seriell erschienen in den Jahren 1989 bis 1998 und in Buchform gesammelt erstmals 1999,' spielt hauptsächlich im Herbst des Jahres 1888, als der unter dem Namen »)ack the Ripper « bekannt gewordene Serienmörder im Londoner Stadtteil Whitechapel wahrscheinlich fünf Frauen auf bestialische Weise ums Leben brachte - die Identität des Täters ist bis heute nicht geklärt, nicht einmal die genaue Zahl seiner Opfer ist sichergestellt. ${ }^{2}$

Der für Idee und Text stehende Moore schließt sich einer historisch schnell widerlegten These an, die den Täter als William Gull, Leibarzt Königin Victorias, identifiziert und das Königshaus selbst als Auftraggeber mit in die Morde involviert sieht3. Dabei ist sich Moore, wie ein extensiver Anmerkungsapparat zum Comic (Appendix I) klarmacht, über die tönernen Füße seiner Quelle durchaus im Klaren; sie erlaubt ihm hingegen, anhand der Figur eines Wissenschaftlers und der Gegenüberstellung unterschiedlicher Wissensdiskurse den Gegensatz von Fakten und Fiktion zu thematisieren.

\section{Das Werden eines Wissenschaftlergeistes}

Sir William Withey Gull (1816-1890), die historische Persönlichkeit, auf welcher der Protagonist von From Hell basiert, war eine bedeutende Figur der englischen Medizingeschichte des 19. Jahrhunderts. Nach dem Studium der Physiologie, vergleichenden Anatomie, Medizin und Chirurgie an der University of London nahm Gulls akademische Karriere einen steilen Verlauf. Schon vor seiner mit der Goldmedaille ausgezeichneten Dissertation führte sie ihn über die Stationen eines Dozenten für Naturphilosophie, Physiologie und Anatomie sowie eines »Medical Superintendent of the wards for lunatics «, danach zur Professur für Physiologie, Mitglied der Royal Society und diversen Ämtern als praktischer Mediziner bis hin zum Leiter des zentralen Londoner Krankenhauses Guy's Hospital;

\footnotetext{
' Alan Moore u. Eddie Campbell: From Hell. Being a Melodrama in Sixteen Parts. Paddington 1999. Zitiert wird nach dieser Ausgabe mit Angabe von Kapitel- in römischen und Seitenzahl in arabischen Ziffern. Die verwickelte Publikationsgeschichte referiert Campbell in »Comics on the Main Street of Culture.«In: ImageTexT 1.2 (2005), <http://www.english.ufl.edu/imagetext/archives/v1_2/campbell/> (Zugriff am 30.4.2014).

2 Die scherzhaft als > Ripperology< bezeichnete Literatur zu den Morden ist immens und wird als Teil des sie umgebenden Mythos von Moore und Campbell mit thematisiert; vgl. L. Perry Curtis, Jr.: Jack the Ripper and the London Press. New Haven u. London 2001, S. 27-28. Von der heute noch aktuellen Faszination des Falls zeugt u.a. eine stark frequentierte Website wie Casebook: Jack the Ripper <http://www.casebook.org> (Zugriff am 13.9.2014).
}

3 Vgl. Stephen Knight: Jack the Ripper. The Final Solution. London 1976. 
nach seiner Erhebung in den Adelsstand erreichte sie ihren Zenit $1872 \mathrm{im}$ (wohl eher symbolischen) Titel eines Leibarztes des Königin.4

Moore und Campbell stellen den Lebenslauf Gulls im zweiten Kapitel des Comics weitgehend faktengetreu dar. Dabei sticht als Schlüsselszene eine Sequenz hervor, die den sechzehnjährigen Protagonisten im Garten seines zukünftigen Erziehers zeigt, während seine Mutter mit diesem ein Gespräch führt. Der junge Gull entdeckt dort im Gebüsch eine tote Maus, die seine Neugier erweckt, worauf er sein Taschenmesser zückt und das Tier seziert (II 5-6, Abb. 1-6):
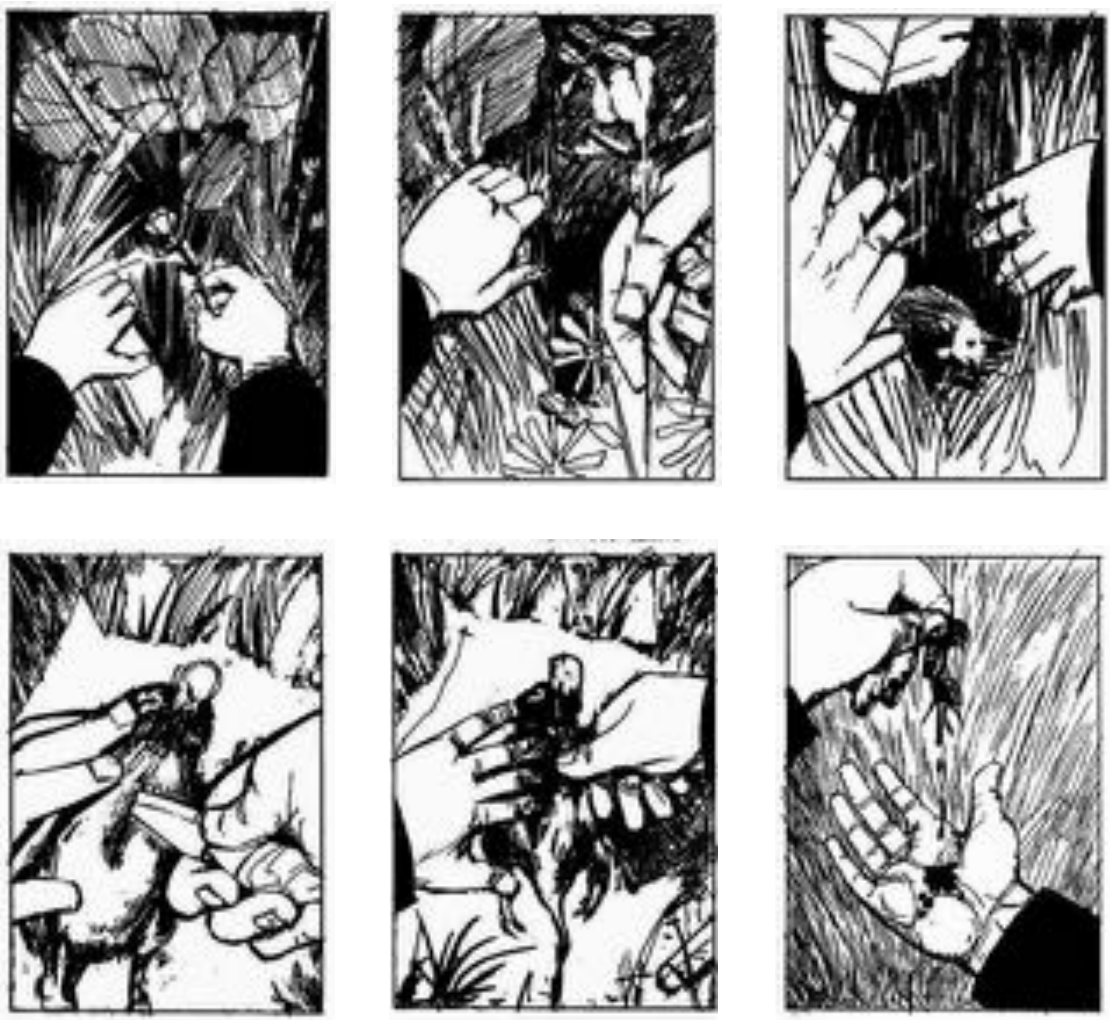

Abb. 1-6 (unterbrochene Panelfolge)

Auffällig an dieser Sequenz ist die deutliche Sexualisierung der Darstellung, die offensichtlich sowohl beim Eindringen in das Gebüsch als auch beim Aufschneiden des Tiers an die Penetration eines weiblichen Körpers gemahnen soll. Moore gibt in der Anmerkung zu den beiden Seiten an, die Szene sei unbelegt, aber durch Gulls ersten Biographen (und Schwiegersohn) Acland inspiriert (vgl. App. I 2), bei dem man denn auch die Bemerkung findet, der junge Gull habe bei Streifzügen durch die Natur allerlei Exemplare der heimischen Tierwelt gesammelt: »The specimens thus obtained [...] were carefully examined and studied with the aid of such books as he could then procure [...], satisfying and yet kindling his longing for knowledge, and opening to him new interests and new views of life.« (Acland 1896) Erscheint die Episode in diesem Licht also als Ausfluss der wissenschaftlichen Neugierde, die erkennen möchte, wie die Welt im Innersten zusammenhält, so eröffnet Eddie Campbell durch die die sprachliche Metaphorik des Zitats (»open-

\footnotetext{
${ }_{4}$ Nach George Thomas Bettany: »Gull, William Withey.« In: Dictionary of National Biography. Hrsg. v. Leslie Stephen u. Sidney Lee. Bd. 23. New York u. London 1890, S. 333-334, sowie Theodore Dyke Acland: William Withey Gull. A Biographical Sketch. London 1896. Eine Würdigung jüngeren Datums, die auch auf die Anschuldigungen Knights eingeht, unternimmt J.M.S. Pearce: »Sir William Withey Gull (1816-1890).« In: European Neurology 55 (2006), S. 53-56.
} 
ing [...] new views of life«) verstärkend aufgreifende visuelle Gestaltung der Szene noch eine weitergehende, historische Perspektive.

\section{Mutter Natur}

Das gewaltsame Eindringen des jungen William Gull in die verborgenen Geheimnisse der Natur führt zurück in die Geburtsstunde der modernen Naturwissenschaft in der frühen Neuzeit, in die Zeit der >naturwissenschaftlichen Revolution< der Renaissance. Ein Zitat Francis Bacons, des Gründervaters der neuzeitlichen Wissenschaftsmethodik, aus dem Jahr 1623 weist wesentliche Übereinstimmungen mit der suggestiv visualisierten Gartenepisode auf:

Neque enim huic rei plus inest negotii, praeterquam ut naturae vestigia persequatis sagaciter, cum ipsa sponte aberret: ut hoc pacto postea, cum tibi libuerit, eam eodem loci deducere et compellere possis. Neque vero praeceperim, ut ex historia ista mirabilium superstitiosae narrationes de maleficiis, fascinationibus, incantationibus, somniis, divinationibus et similibus prorsus exclubantur, ubi de facto et re gesta liquido constet. [...] Ideoque, licet hujusmodi artium usum et praxim merito damnandam censeamus, tamen e speculatione et consideratione ipsarum, si strenue excutiantur, notitiam haud inutilem consequemur, non solum ad delicta in hoc genere reorum rite dijudicanda, sed etiam ad naturae secreta ulterius rimanda. Neque certe haesitandum de ingressu et penetratione intra hujusmodi antra et recessu, si quis sibi unicam veritatis inquisitionem proponat [...].5
Denn bei dieser Sache hat man nichts mehreres zu tun, als daß man die Spuren der Natur emsig nachspüre, wenn solche von selbst verirret, um sie an den Ort zu bringen, wohin man sie haben will. Ich will aber auch nicht gesagt haben, daß aus dieser Geschichte der Wunder, die abergläubische Erzehlungen von Vergiftungen, Bezauberungen, Besizungen, Träumen, Prophezeyungen, und dergleichen, gänzlich wegbleiben sollen [...]. Und diesfalls werden wir, wenn gleich die Anordnung und Anwendung dieser zauberischen Künste zur Bosheit verdammlich ist, dennoch von der Spekulation und scharfen Betrachtung derselben keine unnüze Kenntnis erlangen, als die [...] anderen auch zur weiteren Eindringung in die Geheimniße der Natur wirklich dienen wird. Auch ist wahrhaftig nicht an dem Eintritt [penetratio] und dem Durchdringen in diese verschloßene Pläze [antra = Körperöffnungen ...] zu zweifeln, wenn man sich einzig die Untersuchung der Wahrheit vornimmt [...]. ${ }^{6}$

Spricht schon der Beginn der zitierten Passage von einem gewaltsamen Zugriff, so kommt am Ende die sexualisierte Prägung deutlich zum Vorschein: Die Suche nach der Wahrheit, nach Erkenntnis, wird mit der Penetration eines Körpers gleich-gesetzt. Metaphern von der Weiblichkeit der Natur, in die gewaltsam eingedrungen werden soll, finden sich bei Bacon und anderen Denkern seiner Zeit sehr häufig; die Wissenschaftshistorikerin Carolyn Merchant hat diese Vorstellungen mit der Tendenz der neuzeitlichen Naturwissenschaft zur gewaltsamen Ausbeutung, zum rücksichtslosen Eingriff in die Natur und zu ihrer damit einhergehenden Zerstörung in Verbindung gebracht.7

Eine der gleichen Zeit entstammende allegorische Darstellung aus Michael Maiers Traktat Atalanta fugiens (1618), die den (männlichen) Naturforscher auf den Spuren der als Frau vorgestellten Natur zeigt (Abb. 7), kann diese Deutung stärken: Das begleitende

\footnotetext{
5 Francis Bacon: De Dignitate et Augmentis Scientiarum Libri IX [1623]. Hg. v. Philipp Mayer. Bd. 1. Nürnberg 1829, S. 92-93 (Hervorhebungen vom Vf.).

6 ders.: Über die Würde und den Fortgang der Wissenschaften. Hg. v. Johann Hermann Pfingsten. Pest $1783, \mathrm{~S}$. 176-177 (auch hier Hervorhebungen vom Vf.).

7 Carolyn Merchant: Der Tod der Natur. Ökologie, Frauen und neuzeitliche Naturwissenschaft. München 1994.
} 
Epigramm identifiziert mit den Worten: »Die Vernunfft sey dein Stab ${ }^{8} 8$ eines seiner Instrumente als die Ratio selbst, wobei der phallische Aspekt dieses Attributs durchaus mit Bacons Metaphorik übereinstimmt; die optischen Hilfsmittel Brille und Blendlaterne sollen aufklärerisches Licht ins Dunkel der begehrten weiblichen Geheimnisse und Verstecke bringen.

Bemerkenswert ist, dass beide Belege auch noch ältere Wissensdiskurse weiterführen: Liegt beim Alchemisten und promovierten Mediziner Maier die Mischung aus Okkultismus und naturwissenschaftlichem Interesse auf der Hand, 9 ist es bei Bacon das Zugeständnis, »abergläubische[n] Erzehlungen von

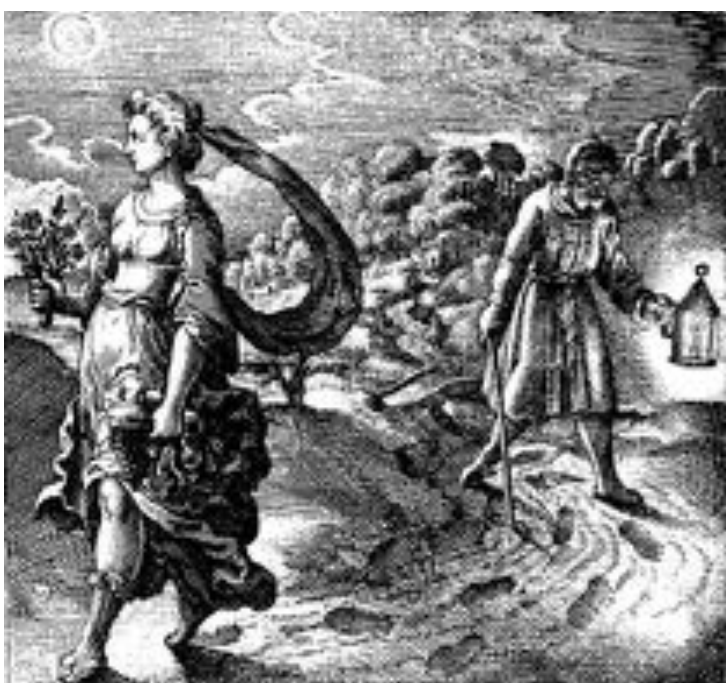

Abb. 7 [...] Träumen, Prophezeyungen, und dergleichen« (a.a.O., S. 176) einen Erkenntnisgewinn nicht völlig abzusprechen.

\section{Eingriffe}

Der mehr oder weniger gewaltsame, penetrierende Eingriff in den (meist weiblichen) Körper wird in From Hell zur, salopp gesprochen, typischen Handbewegung William Gulls, die im Verlauf des Comics wieder und wieder auftaucht. In seiner Funktion als »Medical Superintendent of the wards for lunatics « nimmt er in einer biographischen Episode eine mit wenig Feingefühl oder gar Sympathie durchgeführte vaginale Untersuchung einer psychisch kranken Patientin vor, in deren Verlauf er abfällige Bemerkungen über seine Schutzbefohlenen macht; Campbell inszeniert dies perspektivisch in geradezu obszön wirkender Direktheit (II 11, Abb. 8). Andere Beispiele finden sich in den zahlreichen Darstellungen von Sektionen, unter denen besonders die Entnahme des Herzens eines soeben Verstorbenen heraussticht, bei der das Interesse der Forschung für den Mediziner Gull schwerer wiegt als der explizite Wille und die Gefühle der direkt danebensitzenden Angehörigen (II 22, Abb. 9-10);

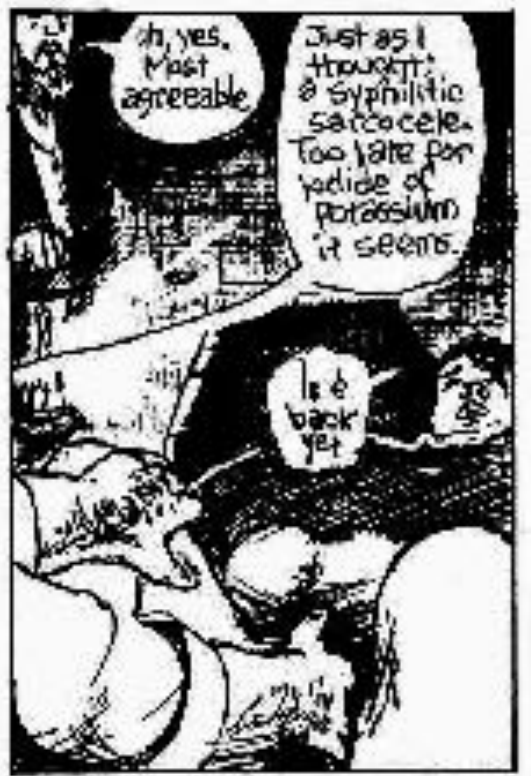

Abb. 8 auch hier suggeriert die Wahl der Perspektive und des Bildausschnitts Distanziertheit und wissenschaftliche Objektivität. Der Comic zeichnet derart das Bild eines kühlen Rationalisten, der seine wissenschaftlichen Ziele ohne Rücksicht auf Emotionen anderer verfolgt.

\footnotetext{
${ }^{8}$ Michael Maier: Atalanta fugiens, hoc est, Emblemata nova de secretis naturae chymica. Faks.-Dr. d. Oppenheimer Orig.-Ausg. von 1618. Kassel 1964, S. 176 (Emblem XLII).

9 Vgl. Ulrich Neumann: »Maier, Michael«. In: Neue Deutsche Biographie. Hg. von der Historischen Kommission bei der Bayerischen Akademie der Wissenschaften. Bd. 15. Berlin 1987, S. 703-704.
} 

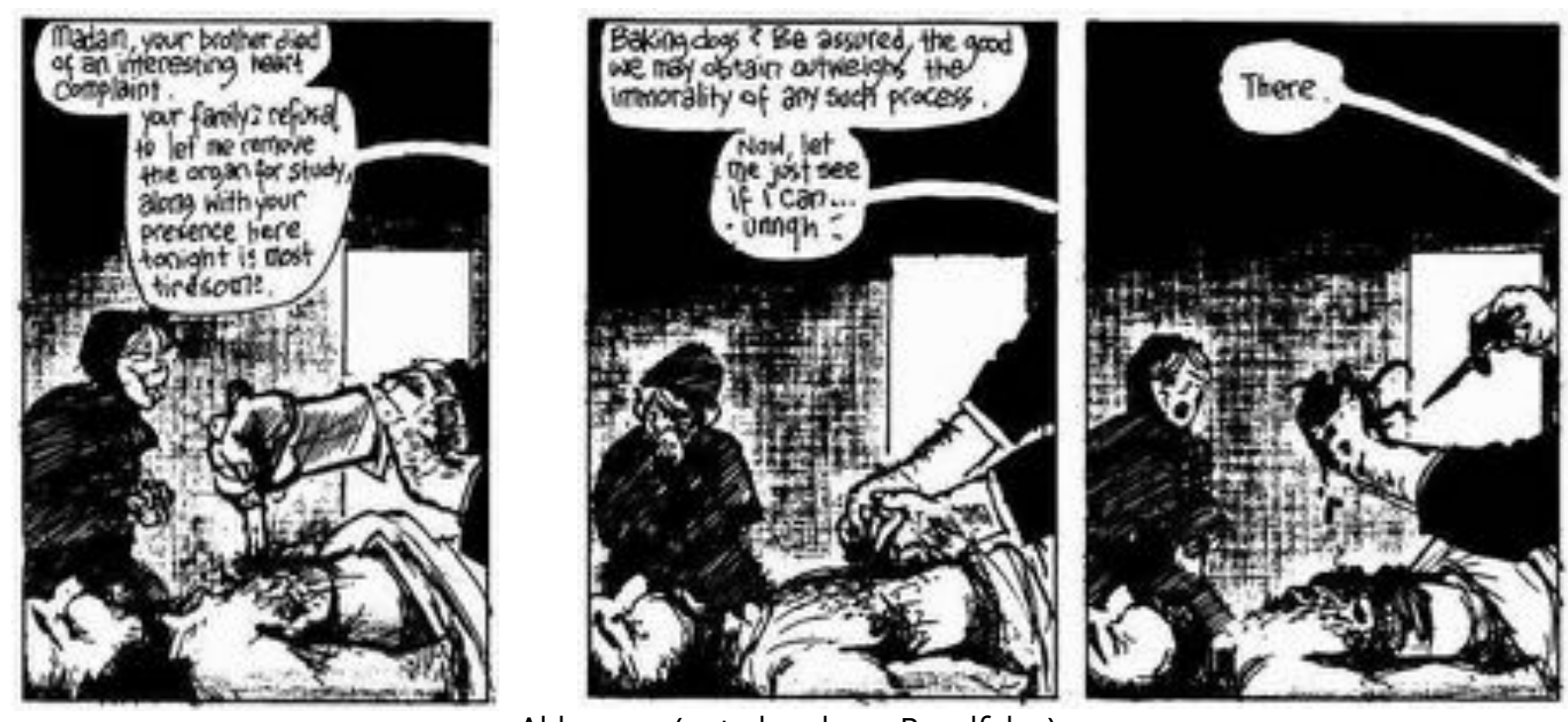

Abb. 9-10 (unterbrochene Panelfolge)

Ganz neue Dimensionen nimmt diese Geste an, wenn Gull seine Mordserie beginnt. Moore und Campbell knüpfen an die nun etablierte Metaphorik an und vertiefen sie gleich beim ersten Opfer, dem der Täter in Übereinstimmung mit den historischen Quellen die Gebärmutter entnimmt. Gull begleitet dies mit der einleitenden Formel des Naturforschers »Let's see what we have h...«, und endet nach einem Blick auf das freigelegte Organ in einer Epiphanie, die ihn sprachlos macht: »It's ... What is it? I've never seen anything quite like ...« Die Vision gipfelt in einer Quintessenz, die die Metaphorik von Sehen und Licht absolut setzt: »Light, Netley. Did you see? She was full of light.« (V 32-33, Abb. 11-12)

Diese erste Leiche ist der Auftakt zu einer Reihe von Morden, bei denen Gull seine Opfer zunehmend schrecklicher verstümmelt und die entnommenen Organe in tranceartigen Ritualen um die Körper herum drapiert. Der erfolgreiche Wissenschaftler und, in der Version des Comics, hochrangige Freimaurer, in deren Reihen ein Jahrhundert zuvor Lessing und Herder, Fichte und Goethe zu finden waren, erscheint dabei als eine Art pervertierte Figur der Aufklärung, getreu nach Horkheimers und Adornos Diagnose, die auf die Spitze getriebene Aufklärung schlage in Mythos und damit in Barbarei um ${ }^{10}$.

\section{Anatomisches Theater}

Durch ein beziehungsreiches Bildzitat wird Gulls Tätigkeit explizit in einen wissenschaftshistorischen Kontext gesetzt. Nach dem vierten Mord erscheint sein Kutscher und Mithelfer Netley in der Privatwohnung des königlichen Leibarztes, um ihm eine dringliche Mitteilung zu machen. Während des Gesprächs ist hinter Netleys Rücken ein Ausschnitt eines an der Wand hängenden Drucks zu sehen, der im darauffolgenden Panel zentral in den Blick genommen wird (IX 16, Abb. 13). Es handelt sich dabei um den letzten Stich des Zyklus The Four Stages of Cruelty (1751) von William Hogarth, mit dem Titel The Reward of Cruelty.

Das Bild zeigt die im Kreise von Medizinern durchgeführte öffentliche Sektion eines hingerichteten Verbrechers, der auf diese Weise noch nach seinem Tode eine zusätzliche Strafe erleiden soll. Die Szene ist der klägliche Abschluss des Lebenslaufs von Tom Nero, dem eigentlichen Protagonisten des vierteiligen Zyklus, der dessen Laufbahn vom ju-

\footnotetext{
${ }^{10}$ Max Horkheimer u. Theodor W. Adorno: Dialektik der Aufklärung. Philosophische Fragmente. Frankfurt/M. 1978.
} 

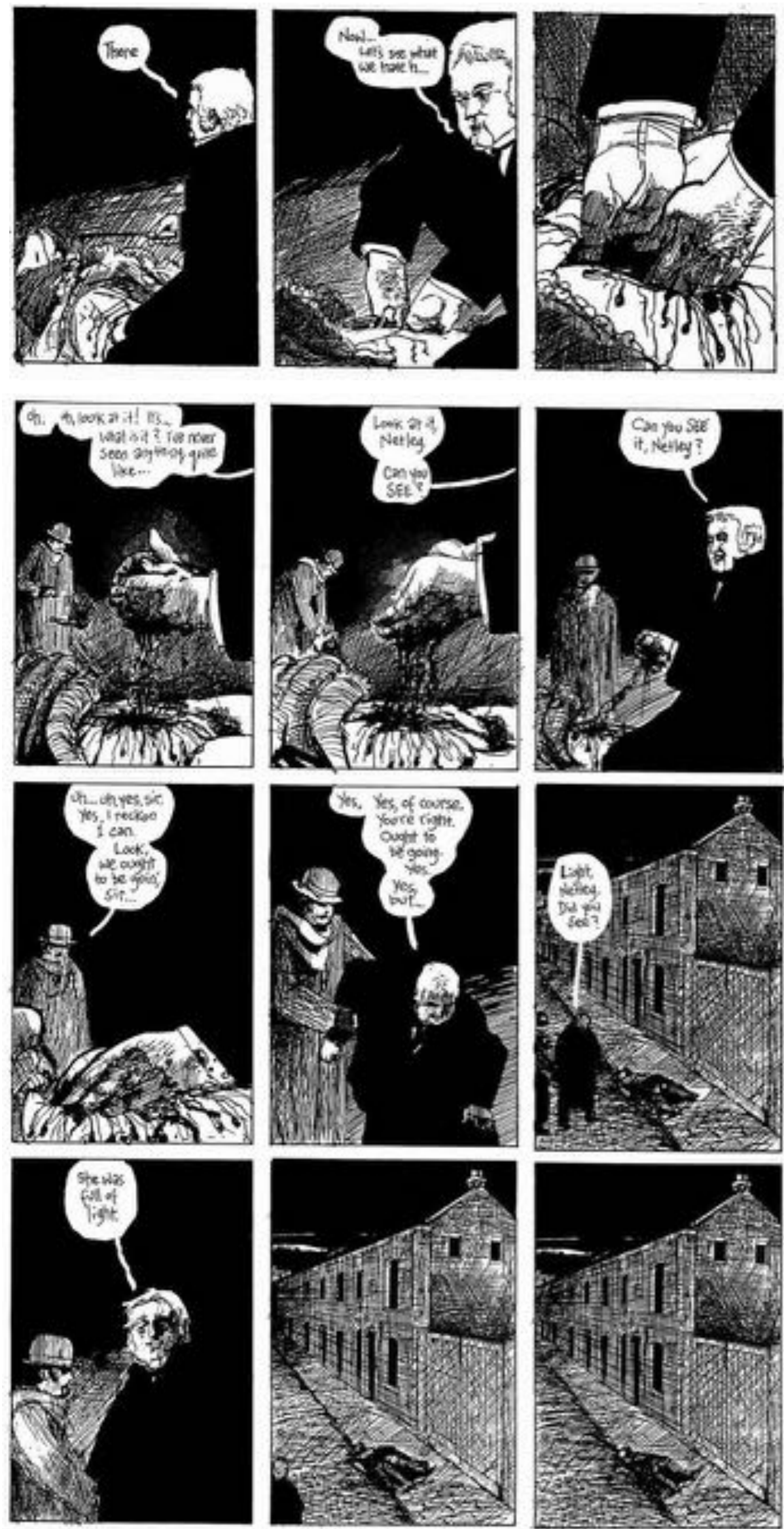

Abb. 11-12 (unterbrochene Panelfolge) 
gendlichen Tierquäler bis hin zum Mörder als abschreckendes Beispiel in Wort und Bild schildert;" eine unausgesprochene Pointe wird ersichtlich im dritten, nicht gezeigten Stich Hogarths, in dem Neros Opfer als grässlich verstümmelte Frauenleiche im Vordergrund an die Opfer Jack the Rippers gemahnt.

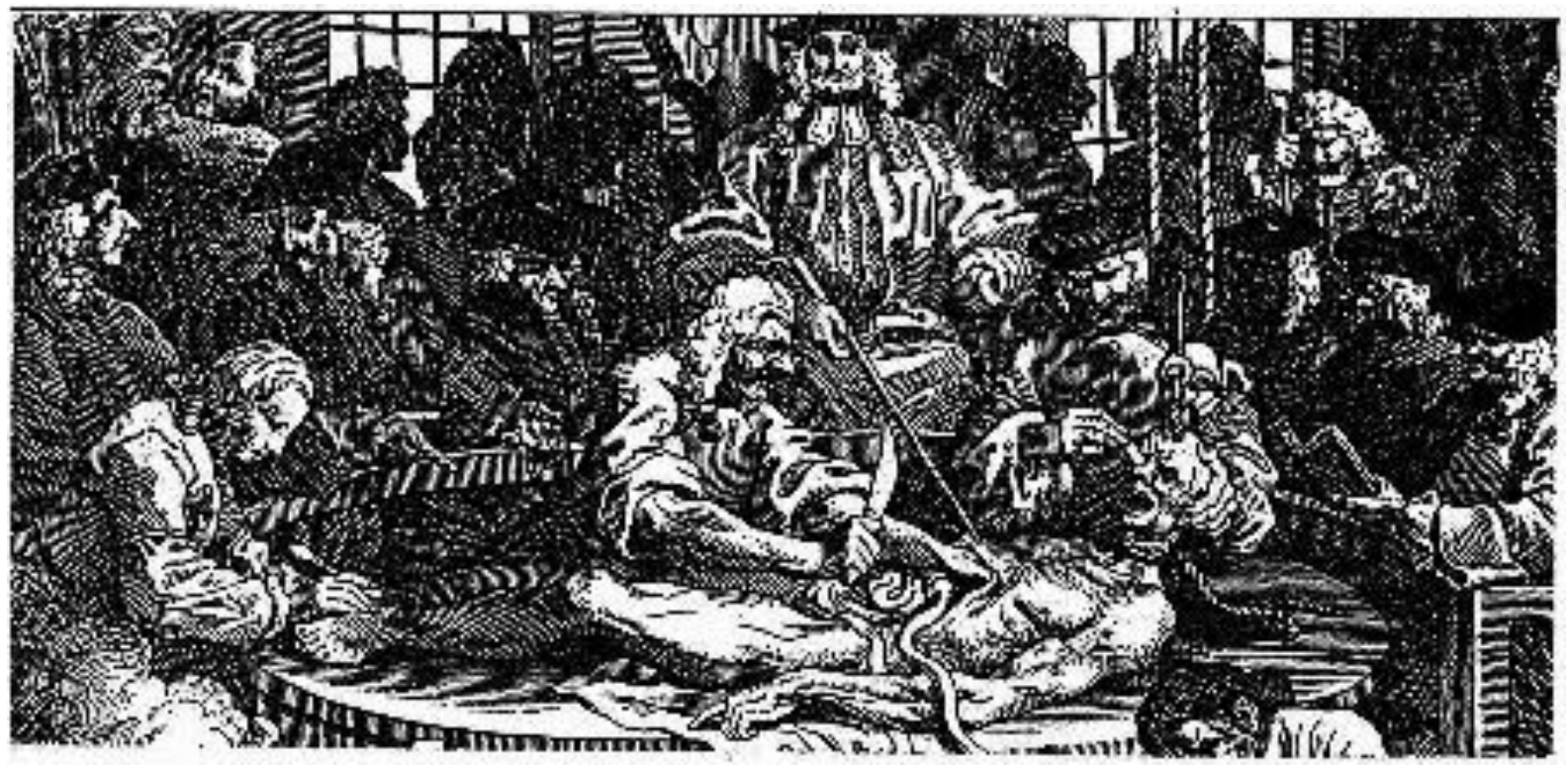

Abb. 13

Die Sektionsszene Hogarths hat mit einiger Wahrscheinlichkeit ein direktes Vorbild im Werk einer weiteren Portalfigur der modernen Naturwissenschaft, nämlich Andreas Vesalius, der mit seinem Durchbruchswerk der modernen Medizin, De humani corporis fabrica libri septem, 1543 den ersten Anatomieatlas auf empirischer Grundlage vorlegte; sein Frontispiz weist eine große Ähnlichkeit mit Hogarths Darstellung auf12 (Abb. 14).

Hartmut und Gernot Böhme haben der Anatomie, durchaus im Einklang mit Carolyn Merchants Befund, geradezu eine Leitfunktion bei der Entwicklung der modernen Rationalitätsstrukturen zugesprochen: »Es ist anzunehmen, daß [...] die Anatomie für die sich entwickelnde Naturwissenschaft die methodologischen Richtlinien gesetzt hat: Sichtbarmachen, Aufschneiden, Entdecken [sic].«13 Symptomatisch erscheint in den oben gezeigten Bildern, dass sowohl bei Hogarth als auch in Vesalius' Frontispiz ein phallischer Zeigestock zum Einsatz kommt, der an denjenigen in Maiers Emblem des Naturforschers (Abb. 7) erinnert und das Seziermesser vorwegnimmt, mit dem Gull seine Morde durchführt.

Hogarths karikierende Darstellung der die Sektion ausführenden Chirurgen wirft letztlich ein negatives Licht auf den gebildeten Mediziner Gull, in dessen Empfangszimmer es hängt: »Hogarth seemed to share the popular belief that surgeons were, on the whole, dis-

\footnotetext{
$"$ Das Bildzitat Hogarths, der verschiedentlich als Vorläufer des modernen Comics gehandelt wird (vgl. z.B. Hermann Josef Schnackertz: Form und Funktion medialen Erzählens. Narrativität in Bildsequenz und Comicstrip. München 1980 oder Thierry Smolderen: Naissances de la bande dessinée. De William Hogarth à Winsor McCay. Paris 2009), ist nur einer der zahlreichen selbstreflexiven Einschläge in From Hell.

12 Da Vesalius' anatomisches Werk im 18. Jahrhundert auch gerne von Künstlern als Vorlage genutzt wurde, hält es Fiona Haslam für möglich, dass es Hogarth zugänglich war (vgl. ihre Studie From Hogarth to Rowlandson. Medicine in Art in Eighteenth-century Britain. Liverpool 1996, S. 258-259).

13 Böhme, Hartmut und Gernot: Das andere der Vernunft. Zur Entwicklung von Rationalitätsstrukturen am Beispiel Kants. Frankfurt/M. 2. Aufl. 1992, S. 52. Interessanterweise weisen die beiden Autoren im Übrigen darauf hin, dass die führende Stellung der Anatomie zumindestens teilweise »durch die gesellschaftliche Rolle der Medizin als Gerichtsmedizin bestimmt« sei (ebd.).
} 


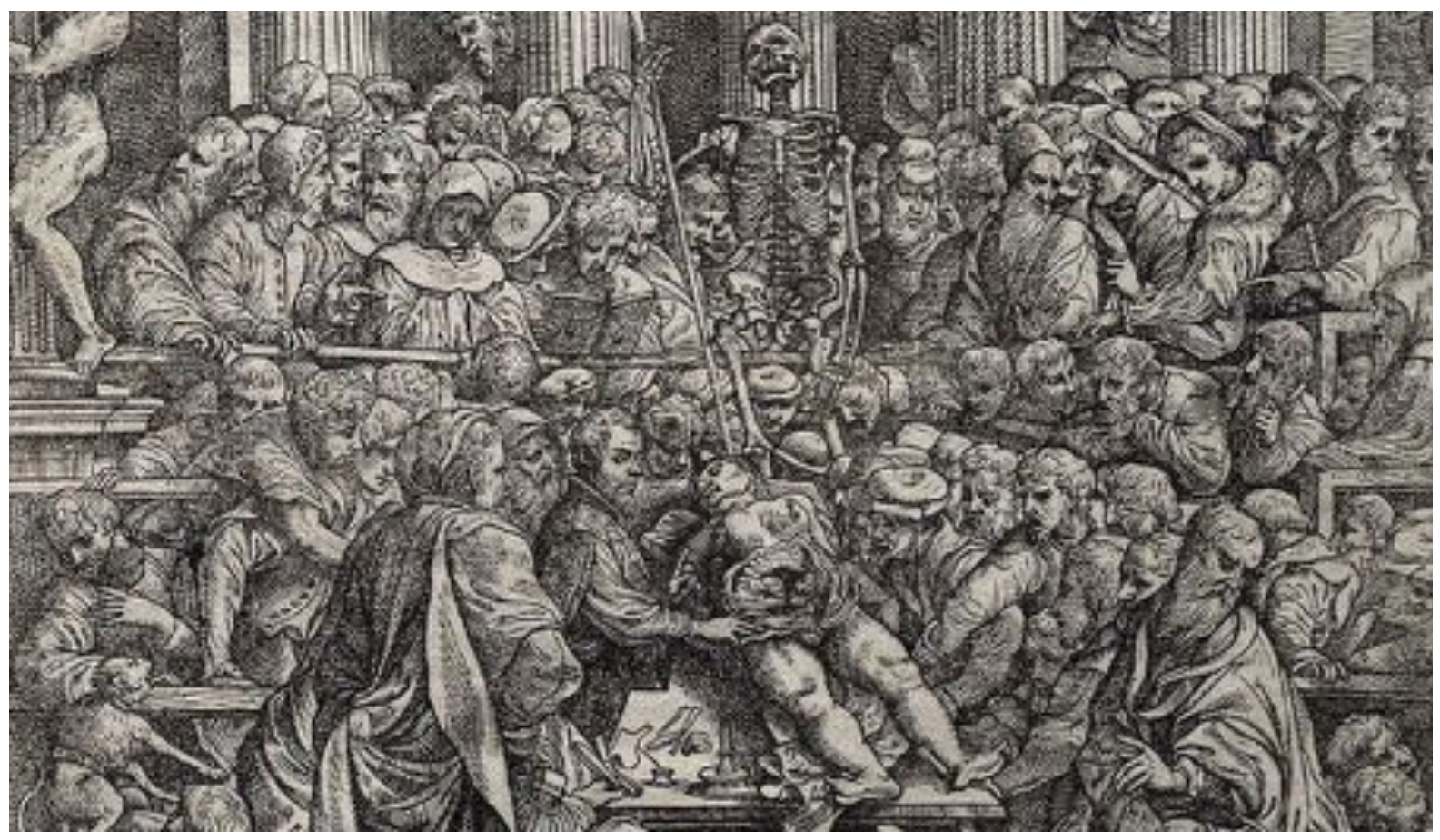

Abb. 14 (Ausschnitt)

reputable, insensitive to human suffering and prone to victimise people in the same way that criminals victimised their prey.« (Haslam 1996, S. 257)

In From Hell wird Gull nur an einer Stelle bei einer Lehrtätigkeit gezeigt: Als er sein letztes Opfer in einer stundenlangen brutalen Blutorgie buchstäblich zerstückelt (historische Photos des Leichenfundorts zeigen ein schier ungeheuerliches, schwer erträgliches Ausmaß an Verstümmelungen), wird dies in Moores und Campbells Inszenierung mehrfach durch unterschiedliche Visionen unterbrochen, und bei einer dieser Gelegenheiten imaginiert sich Gull als Dozent im anatomischen Theater (X 14, Abb. 15). Ironischerweise sind die in den Zuschauerreihen sichtbaren Schüler alle als spätere englische Serienmörder identifizierbar ${ }^{14}$ - Jack the Ripper erzieht sich hier seine eigenen Nachfolger.

\section{Scharlatanerie}

Der Gegenspieler des Wissenschaftlers Gull ist in From Hell Robert Lees, ein ebenfalls historisch belegter englischer Spiritualist und Schriftsteller (1849-1931). Moore stützt sich hier auf der Quellenlage nach wenig haltbare Gerüchte, nach denen Lees als Medium für Königin Victoria den Geist ihres verstorbenen, über alles geliebten Gatten heraufbeschworen haben soll, 15 und nutzt dies, um im Buckingham Palast ein unverhofftes Zusammentreffen mit Gull in Szene zu setzen. Lees zeigt sich hocherfreut über die Gelegenheit, den berühmten Leibarzt quasi auf kollegialem Terrain kennenzulernen, aber der Rationalist Gull fertigt ihn kühl mit dem Vorwurf ab, er sei ein Scharlatan, »profiting from delusions born of bereavement « (IX 12). Dies nagt an dem derart Gescholtenen, so dass er aus Rache bei der Polizei angibt, er habe durch seine Visionen Erkenntnisse über die Identität von Jack the Ripper erhalten, untermalt durch einen theatralisch vorgetäuschten Anfall spiritueller

\footnotetext{
14 Vgl. Alan Moore u. Eddie Campbell: The From Hell Companion. Marietta 2013, S. 226.

15 Vgl. Jennifer D. Pegg: »Robert James Lees. Visions From Hell? « In: Ripperoo 11

<http://www.casebook.org/dissertations/ripperoo-lees.html> (Zugriff am 3. Mai 2014).
} 
Trance: »A-and the KILLER. I could SEE him. H-he was a doctor. He killed those women with a doctor's knife.« Als Quelle seiner Eingebungen spezifiziert er ein weiteres Phänomen älterer, auch von Bacon genannter Wissensdiskurse: »These fearful waking dreams.« (XII 6) Nachdem er den leitenden Ermittler Inspector Abberline zu Gulls Wohnung geführt hat, gesteht dieser seinen Besuchern auf der Stelle, zu Lees eigener Verblüffung, die Ripper-Morde begangen zu haben.

Lees begegnet dem Leser von From Hell schon weit vor diesen Ereignissen, auf den ersten Seiten des Buches im 1923 spielenden Prolog. Dort gesteht er im Gespräch mit Abberline über die Zeiten der Ripper-Morde, dass alle seine Visionen, Trancen und Voraussagen nur vorgetäuscht waren (Prolog 3-5, Abb. 15-16).
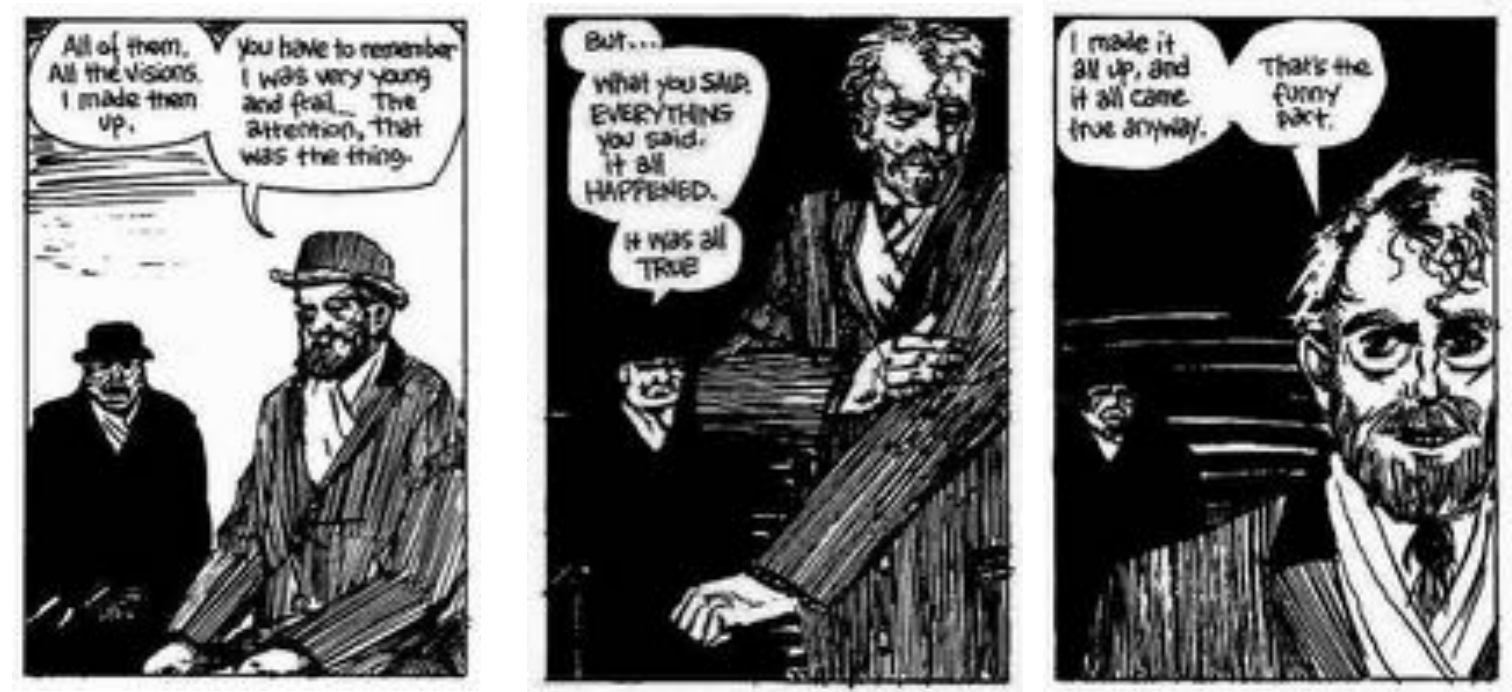

Abb. 15-16 (unterbrochene Panelfolge)

Lees erscheint damit schon von vornherein als Lügner und Betrüger und wirft somit die alte Frage nach dem Verhältnis von Fakten und Fiktion, Lüge und Wahrheit auf, die seit Platon der Literatur und Kunst gestellt wird. Moore thematisiert dies auch im Anmerkungsapparat des Comics, der dem umfangreichen Werk zusätzlich zur Zurückhaltung bei der Verwendung comicspezifischer Erzählmittel (u.a. Vermeidung von Onomatopöien, Bewegungslinien, Farbe oder karikierender und simplifizierender Darstellung) eine dokumentarische Anmutung verleiht: »The dialogue between the two men is thus a fabrication: Lees always claimed his psychic abilities were perfectly genuine, and the confession I have attributed to him here is based upon my own intuitions and prejudices.« (App. I 1)

Der Nachsatz Lees im letzten zitierten Panel gibt diesem nonchalanten Eingeständnis einer licentia poetica allerdings eine überraschende Wendung: »l made it all up, and it all came true anyway.«

\section{»Prophezeyungen«}

Bacons »abergläubische Erzehlungen von [...] Träumen, Prophezeyungen, und dergleichen « sind ein prominenter Einschlag in From Hell; Lisa Coppin hat dies mit Freuds Konzeption des Unheimlichen in Verbindung gebracht. ${ }^{16}$ Dabei sticht eine Szene hervor, in der Inspector Abberline eine amerikanische Schaustellertruppe verhört - versprengte Reste der

\footnotetext{
${ }^{16}$ Lisa Coppin: »Looking Inside Out. The vision as particular gaze in From Hell (Alan Moore \& Eddie Campbell).« In: Image [QJ] Narrative 3.1 (2003), <http://www.imageandnarrative.be/inarchive/uncanny/lisacoppin.htm> (Zugriff am 1.5.2014).
} 
kurz zuvor in London vor Mitgliedern der königlichen Familie aufgetretenen WildwestShow von William Cody alias Buffalo Bill -, deren Anführer einen prophetischen Traktat zitiert (VI, 15, Abb. 17):
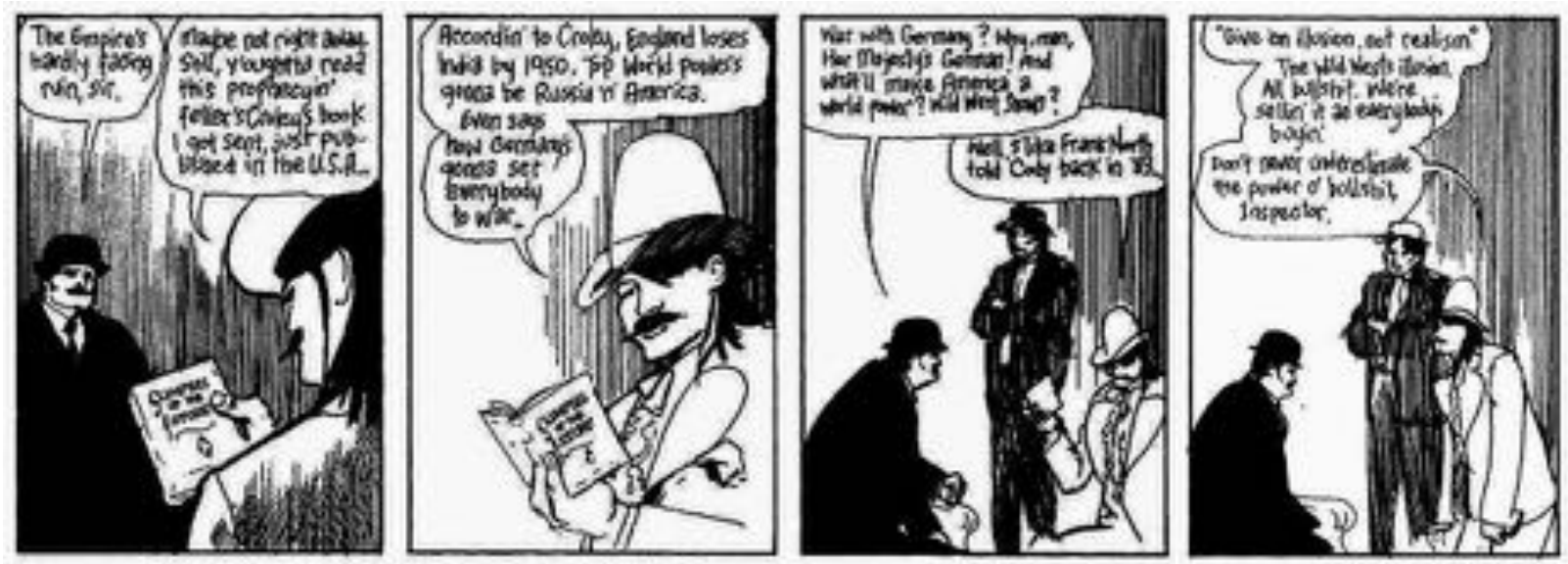

Abb. 17

Es ist wohl naheliegend, »the power of bullshit« als Macht der Fiktion und der Phantasie zu deuten - das 1888 erschienene Bändchen Glimpses of the Future des amerikanischen Journalisten David Goodman Croly enthält in der Tat eine erstaunliche Trefferzahl an Vorhersagen 17 - »and it all came true anyway«.

Der erstaunlichste Traum jedoch wird in der einzigen nicht in England spielenden Passage des Comics erzählt: Unter der Orts- und Zeitangabe »Braunau, Upper Austria, August 1888 « wird das fünfte Kapitel von drei Seiten eingeleitet, die ein im Original deutsch sprechendes Paar beim Liebesakt zeigen; auf dem Höhepunkt schreckt die Frau unter dem Eindruck einer unheimlichen Vision zusammen (V, 1-3, Abb. 18): »lch ... ich habe eine ERSCHEINUNG gehabt ... einen schrecklichen Traum. / Es war eine Kirche, eine böse Kirche. Es war in einem Ort voller Juden. // Und ... die Türen sind aufgeplatzt, und es strömte überall heraus. / All die Leute, all die Juden ... es hat sie alle einfach weggewaschen.«
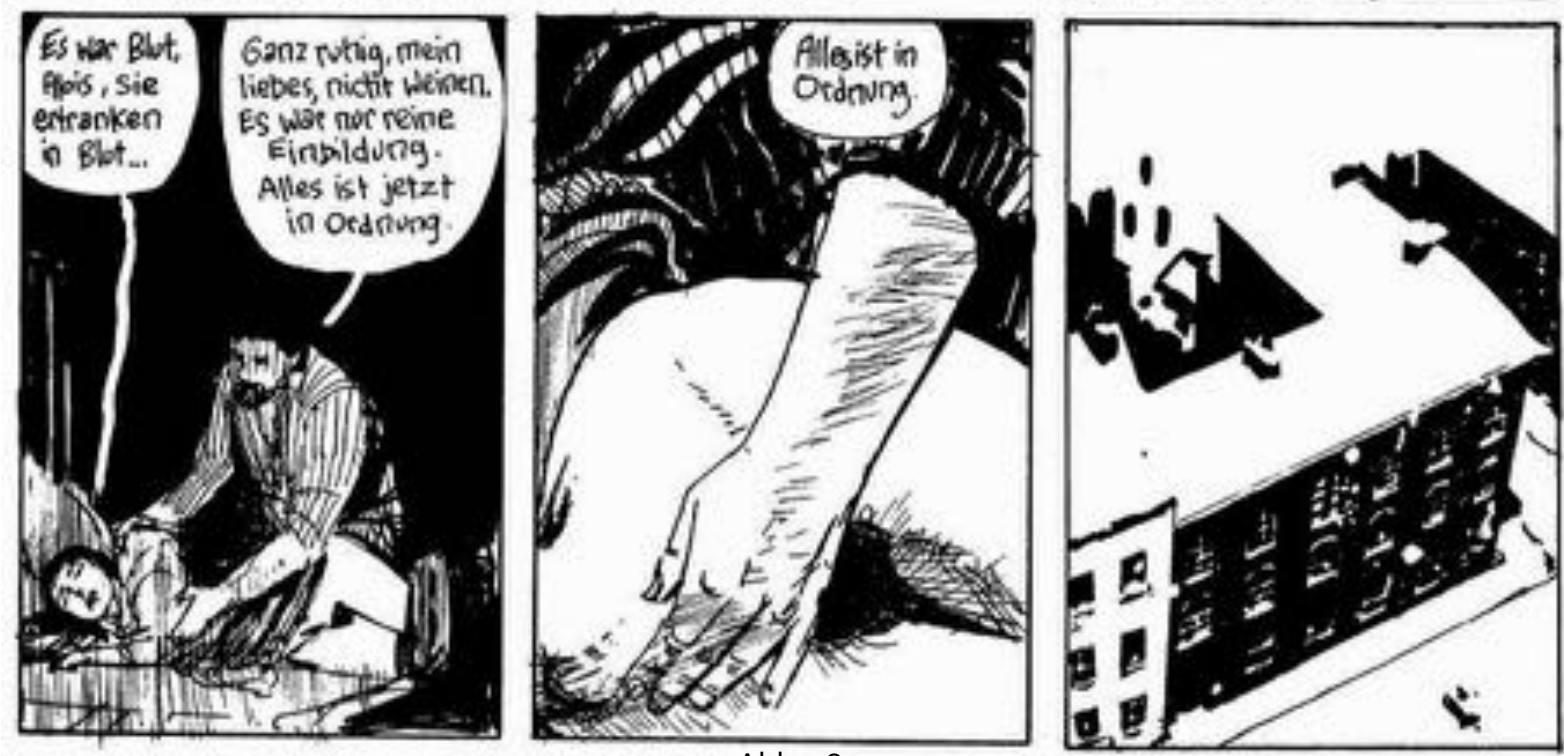

Abb. 18

Kein Zweifel: Aus dem so zärtlich gehaltenen Schoß wird neun Monate später der am 20. April 1889 geborene Adolf Hitler kriechen, der die halbe Welt mit Blut überziehen und

17 David Goodman Croly: Glimpses of the Future. Suggestions as to the Drift of Things. New York u. London 1888. 
dabei Millionen Juden seinfach wegwaschen< wird. Damit eröffnen Moore und Campbell eine weitere Perspektive auf die prophetischen Szenen des Comics.

\section{Eingeweideschau}

Dem Rationalen scheinbar entgegengesetzt, stoßen ältere Wissensdiskurse wie Mantik, Traumdeutung, Magie und okkulte Praktiken in der neueren Forschung nicht mehr auf pauschale Abwertungen. »Das Mantische liegt dem Wissen nicht unvordenklich voraus, sondern stellt selbst eine hochgradig reflektierte Denkform dar. «18 Im Licht dieser Erkenntnisse lässt sich in der oben beschriebenen Schlüsselszene vom Forscherdrang des jungen William Gull wie auch in seiner späteren Metamorphose zu Jack the Ripper noch eine andere Dimension ausmachen.

Der Organe entnehmende Griff kann als Zugriff des neuzeitlichen wissenschaftlichen Forschungsdrangs gelesen werden, aber gleichzeitig auch als die Geste des Hieroskopen, der eine Eingeweideschau vornimmt. Die Hieroskopie oder Mantik war eine in der Antike im gesamten Mittelmeerraum und in Vorderasien vielfach bezeugte und elaborierte Methode, aus den inneren Organen von Opfertieren, vor allem der Leber, die Zukunft vorauszusagen:

Die hochentwickelten Lehren von der Zeichenhaftigkeit der Welt galten in der klassischen Antike als eine große Errungenschaft des menschlichen Geistes, dem es im Lauf der Zeiten gelungen war, in Natur, Umwelt und Gesellschaft verläßliche Hinweise auf Kommendes ausfindig zu machen, und überdies auch die Gesetzmäßigkeiten zu erkennen, die die Zeichen der Warnung und Leitung mit einem zukünftigen Geschehen verbanden. [...] Die Disziplinen, die sich ernsthaft um Einsicht in die Zukunft bemühten, waren [...] geprägt von unbestechlichem Erkenntniswillen und aufrichtigem Forscherdrang, von sorgfältigem Beobachten und exaktem Dokumentieren, von scharfsinnigem Denken und kühnen Theorien und deshalb, nicht anders als unsere Wissenschaften, hochgeachtet und von großer Autorität. ${ }^{19}$

Versteckte Hinweise auf diese lange Tradition finden sich wiederum während der Zerstückelung von Mary Kelly, des letzten Ripper-Opfers, als Gull während der Szene im anatomischen Theater längere Zeit über die Leber doziert $(X$ 14, Abb. 15 oben) und als dann schließlich er selbst plötzlich für kurze Zeit als altorientalische mythologische Figur dargestellt wird und diese Mythen auch benennt (X 19, Abb. 19).

Aber es bleibt nicht nur bei diesen versteckten Hinweisen, denn das eigentliche Ziel der Morde Gulls sind Visionen, die ihm buchstäblich die Zukunft zeigen, und zwar diejenige der Gegenwart, der Entstehungszeit des Comics. Bei einer dieser Entrückungen sieht er durch ein erleuchtetes Fenster einen Mann neben einem Fernsehgerät (VII 24), später wächst vor seinen Augen ein moderner Wolkenkratzer aus dem Boden
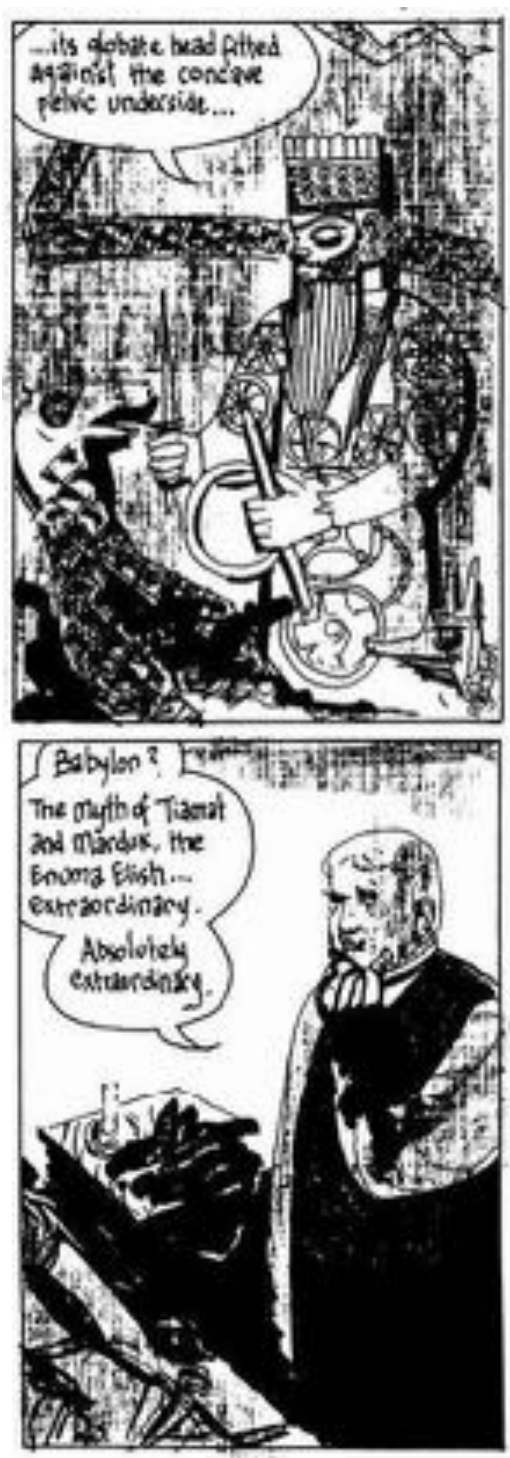

Abb. 19

\footnotetext{
${ }^{18}$ Guido Kreis: »Eine unmittelbare Erfahrung.« In: Mantik. Profile prognostischen Wissens in Wissenschaft und Kultur. Hrsg. v. Wolfram Hogrebe. Würzburg 2005, S. 37-55, hier: S. 54.
}

19 Stefan M. Maul: Die Wahrsagekunst im alten Orient. Zeichen des Himmels und der Erde. München 2013, S. 11. 
(VIII 40), und am Höhe- und Schlußpunkt der Mordserie sieht er sich selbst mitsamt der Leiche Mary Kellys in ein mit Kopierern, Computern und Handys ausgestattetes Großraumbüro versetzt (X 20-22).

Neben der magischen Praxis der Eingeweideschau erinnern die Trance-ähnlichen Erfahrungen Gulls an die schamanistischen Praktiken der Geistreise, ${ }^{20}$ an die auch die ausgedehnte Reise des im Sterben liegenden Gulls durch das London verschiedener Zeitebenen anschließen könnte (XIV). Zur gewissermaßen prähistorischen Vorgeschichte der modernen Medizin gehörig, sind Konstrukte dieser synkretistischen religiösen Vorstellungswelt noch in den halb ironisch-imaginativen magischen Konzepten erkennbar, denen sich Moore seit 1993, mitten in der Arbeit an From Hell, verschrieben hat. ${ }^{21}$

Doch als sich Gull inmitten der Büroarbeiter des ausgehenden 20. Jahrhunderts selbst am Ziel seiner Bestrebungen wähnt und sich umschaut, tritt eine völlige Ernüchterung ein. Er hält den Anwesenden eine Moralpredigt, in der er sie - die ihn offensichtlich nicht wahrnehmen - der Gleichgültigkeit und Gefühllosigkeit beschuldigt und ihnen gleichzeitig prophezeit, dass er, Jack the Ripper, ständig bei und in ihnen sei, ihr dunkler Grund:

With all your shimmering numbers and your lights, think not to be inured to history. Its black root succours you. It is INSIDE you. // Are you asleep to it, that cannot feel its breath upon your neck, nor see what soaks its cuffs? // See me! Wake up and look upon me! I am come amongst you. I am with you always! (X 21)

Er sieht damit seine Aufgabe als beendet an (was auch sein bereitwilliges Geständnis erklärt), und es scheint ihm selbst vor dem Resultat zu grauen, wie sich aus seiner Antwort auf Netleys Frage »|s it finished with? « herauslesen lässt: »It is beginning, Netley. Only just beginning. For better or worse, the twentieth century. I have delivered it.« (X 33)

From Hell erweist sich so auch als ein Stück Gegenwartskritik; dass Gull kurz vor diesem resignierten Schlusswort die Asche des verbrannten Herzens von Mary Kelly in den Wind gestreut hat, tut sein Übriges, um die Linie zur Zeugung Adolf Hitlers zurück zu ziehen.

\section{Mad scientist}

Zuvor wird die Figur des Wissenschaftlers William Gull von ihm selbst noch in ganz andere Zusammenhänge gestellt. Wie er selbst Netley auf einer ausführlichen Kutschfahrt durch London zu verstehen gibt, haben seine Morde an den fünf Frauen ursprünglich das Ziel, das Matriarchat zu schwächen und im ewigen Krieg der Geschlechter Partei zu ergreifen dass er den Auftrag dazu von einer Frau, Königin Victoria persönlich, erhält, ist eine ironische Pointe am Rande. Während der auf nicht weniger als 34 Comicseiten geschilderten Kutschfahrt zeigt der auch in der Stadtgeschichte beschlagene Mediziner dem Proletarier Netley London als Schauplatz historischer Auseinandersetzungen zwischen Männern und Frauen, sei es als Spuren kriegerischer Auseinandersetzungen, die in Straßen- oder Platznamen präsent sind, sei es als symbolische Errichtungen im Stadtbild immer noch bestehender phallischer Bauwerke, vor allem Obelisken, Türme und Kirchen, Landmarken dieses beständigen Kampfes.

\footnotetext{
${ }^{20} \mathrm{Vgl}$. Mircea Eliades umstrittene, aber ungemein einflussreiche religionsphänomenologische Studie von 1951: Schamanismus und archaische Ekstasetechnik. Zürich u. Stuttgart 1954; eine neuere Darstellung aus Sicht des Historikers bietet Ronald Hutton: Shamans. Siberian Spirituality and the Western Imagination. London 2001.

${ }^{21}$ Vgl. die Darstellung seines initialen divinatorischen Erlebnisses im Briefwechsel mit Dave Sim: 》Correspondence: From Hell.« In: Alan Moore. Portrait of an Extraordinary Gentleman. Hrsg. v. smoky man u. Gary Spencer Millidge. Leigh-on-Sea 2003, S. 303-345, hier S. 322-324.
} 
Auf der Seite des Mannes stehen dabei »reason, logic, science« (IV 11), die die modernen Wissenschaften fundierende Ratio, symbolisiert durch die Sonne Apollons, während die weibliche Natur durch »magic, art and madness « (ebd.), dionysische Trunkenheit (im Zitat unten durch >Claret<, Bordeaux-Wein, angedeutet) und das Symbol des Mondes gekennzeichnet ist; bezeichnenderweise schließt dies auch den Bereich der Phantasie, Kunst und Dichtung, mit ein:

'Tis in the war of Sun and Moon that man steals Woman's power; that Left Brain conquers Right ... // ... that reason chains insanity. At Bedlam, yonder, I've heard men proclaim they ruled the Eagles; vow they'd war upon the Stars if I'd but give them claret. // Lunatics are soldiers of the Moon, alongside poets, artists, sorcerers, all warring on the stars, which are but distant suns. (IV 21):

Aber in Gulls Systematik schleicht sich eine ihn selbst betreffende Schieflage ein: Nicht nur preist er wiederholt die >dionysische< Abstammung der von ihm präferierten Kirchenarchitektur (vgl. II 13-14, IV 13 u. passim), sondern er selbst ist ständig mit Weintrauben in der Hand zu sehen (IV 23, VIII, $35 \mathrm{u}$. passim); er betäubt seine Opfer mit Trauben, die er mit Laudanumlösung überzieht (vgl. V 22) - ist aber die Weintraube nicht seit jeher ein ikonographisches Attribut des Dionysos, Gott der Trunkenheit und des heiligen Rausches, dieser wiederum der Gegenspieler des Sonnengottes Apollon? Hier spaltet sich Gull auf (IV 22, Abb. 20):
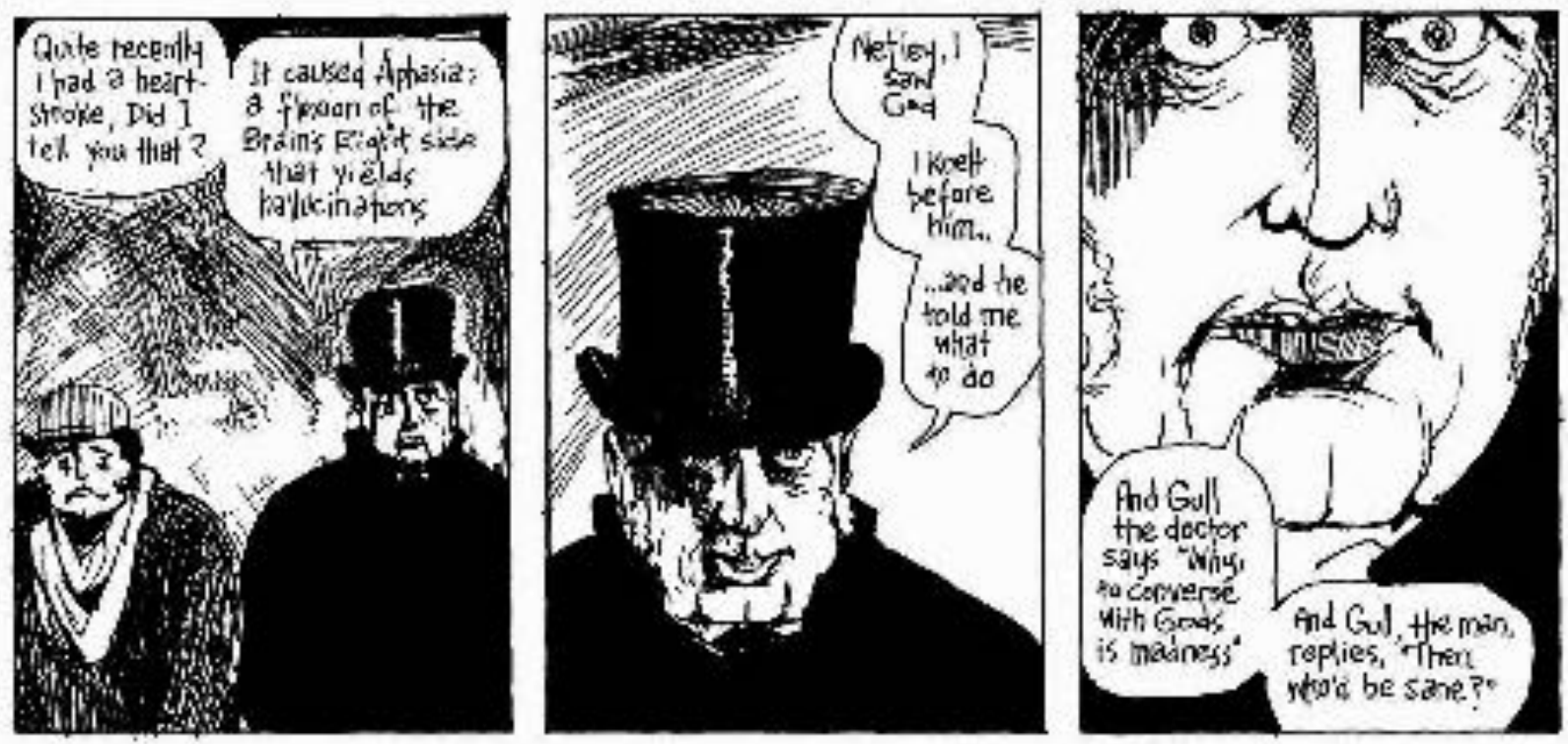

Abb. 20

Der Schlaganfall setzt seine rechte, weibliche Gehirnhälfte in Funktion, und der - schon von Kindesbeinen an ersehnte (vgl. II 3) - Auftrag Gottes (der ihm in Gestalt einer freimaurerischen Dreieinigkeit erscheint, vgl. II 26 u. II 17) signalisiert, dass damit eine höhere Macht in sein Leben getreten ist, aber sein rationaler Skeptizismus (»Gull the doctor «) ist so groß, dass er angesichts des Widerspruchs dem Wahnsinn verfällt.

Gull reiht sich damit ein in die Tradition des mad scientist, die in Literatur, Film und Comic gleichermaßen stark ausgeprägt ist;"22 Misogynie zeigte sich schon im Urbild des Dr. Frankenstein, der die Rolle der Frau bei der Fortpflanzung ersetzen möchte, um die als bedrohlich empfundene Sexualität zu domestizieren. ${ }^{23}$ Das bekannteste Beispiel, bei dem

22 Vgl. dazu Torsten Junge u. Dörthe Ohlhoff (Hrsg.): Wahnsinnig genial. Aschaffenburg 2004.

${ }^{23}$ Vgl. Karin Kaltenbrunner: »Das Labor als Ort des Schreckens. Zur Repräsentation des Wissen-schaftlers im Film.« In: medienimpulse 4 (2011) <http://www.medienimpulse.at/articles/view/368> (Zugriff am 5.5.2014). 
ebenfalls die Aufspaltung eines Wissenschaftlers in zwei widerstrebende Teilaspekte erfolgt, wird in From Hell selbst zitiert: Gull reist am Ende seines Lebens in einer Todesvision durch die Zeiten und trifft dabei auf verschiedene Literaten und Künstler, unter anderem auch auf Robert Louis Stevenson, den er zu seiner populärsten Schöpfung inspiriert (XIV 15, Abb. 21).
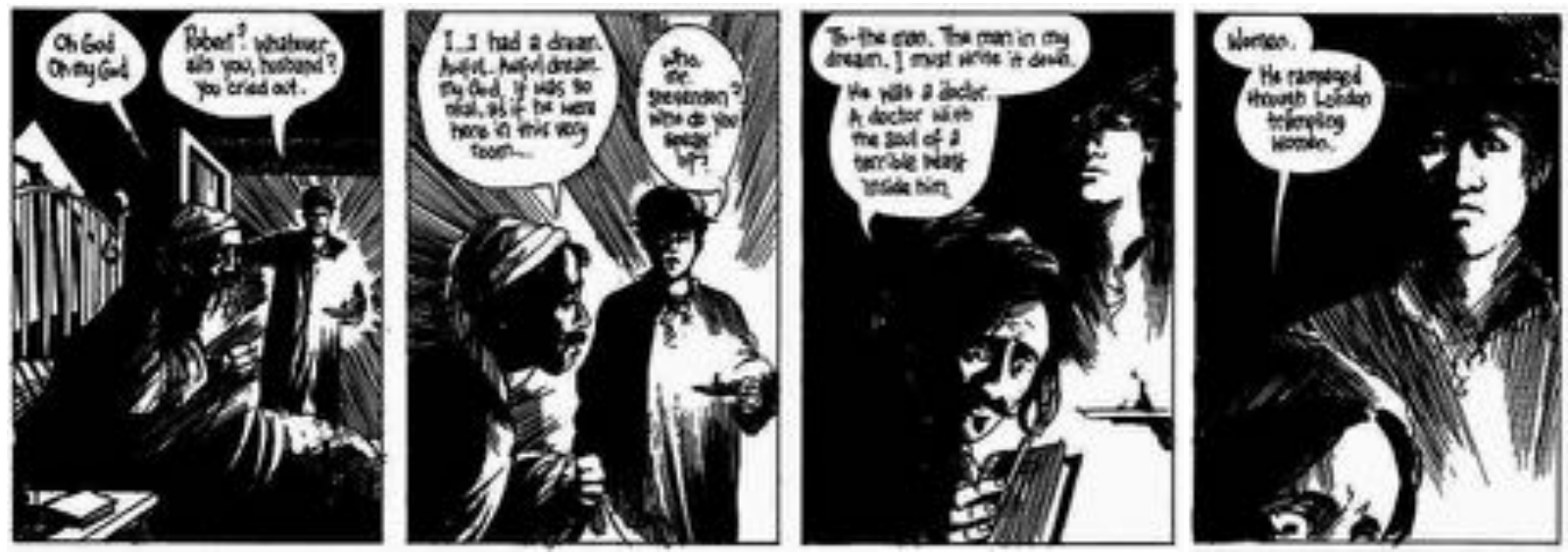

Abb. 21

\section{»What is the fourth dimension?«}

Die Reise durch Raum und Zeit, die William Gull im letzten Kapitel des Comics unternimmt, kann im Zusammenhang von Spekulationen über die >vierte Dimension< gesehen werden, die Moore wiederum im Anschluss an wissenschaftliche Diskurse in From Hell unternimmt. Zum ersten Mal fällt der Begriff in einer Diskussion, die Gull mit seinem Freund James Hinton führt. Dieser erzählt ihm, als er die historischen Dimensionen bestimmter Kirchenarchitekturen erörtert, sein Sohn habe ganz ähnliche Theorien vorgelegt: »They suggest Time is a human illusion. ... that all times CO-EXIST in the stupendous whole of eternity. He hopes to publish a pamphlet one day«, der Titel sei »What is the fourth dimension?«. (II 14)

Vater und Sohn sind historisch verbürgte Persönlichkeiten, den genannten Traktat hat Letzterer, der exzentrische Mathematiker Charles Howard Hinton (1853-1907) 1880 publiziert. Die vierte Dimension, in der Nachfolge Bernhard Riemanns abweichend von der späteren temporalen Konzeption Einsteins als geometrisch-räumliche verstanden, hat Hinton später noch mehrfach abgehandelt, unter anderem 1888 in A New Era of Thought, wo er den Begriff >Tesserakt< für einen vierdimensionalen Würfel prägte, und noch einmal 1904 in der Schrift The Fourth Dimension, in der er Anleitungen zur Entwicklung des mehrdimensionalen räumlichen Vorstellungsvermögens vorlegte. ${ }^{24}$ Bemerkenswert ist daneben noch An Episode of Flatland (1907), die an den bekannten mathematisch fundierten Roman von Edwin Abbott Abbott anschließt. Die kurze Abhandlung »What is the Fourth Dimension?« wurde 1884 mit einer Reihe anderer Aufsätze unter dem Titel Scientific Romances wiederveröffentlicht; wie der Obertitel schon andeutet (auch Abbotts Flatland trug die Genrebezeichnung Romance, also etwa >Liebesroman<), handelt es sich dabei nicht um fach-

24 Vgl. Rudolf v. B. Rucker: »Introduction« [zu] Charles H. Hinton: Speculations on the Fourth Dimension. Selected Writings. Hrsg. v. Rudolf v. B. Rucker. New York 1980, S. v-xix, und Linda Dalrymple Henderson: »Darstellung und Vorstellung der vierten Dimension in der Kunst und Kultur des 20. Jahrhunderts. « In: Zahlen, Zeichen und Figuren. Mathematische Inspirationen in Kunst und Literatur. Hrsg. v. Andrea Albrecht, Gesa von Essen u. Werner Frick. Berlin u. Boston 2011, S. 128-159, zu Hinton hier S. 134-136. 
wissenschaftliche Arbeiten, sondern um Bemühungen, in verständlicher Sprache und teilweise mit fiktionalen Mitteln mathematische Sachverhalte zu popularisieren, was vielleicht zum Teil auch seine Anziehungskraft auf Nicht-Mathematiker erklären kann.

Das Konzept einer vierten Dimension neben den uns bekannten dreien hat ein enormes Echo in Kunst und Literatur gefunden; Hintons Schriften übten direkt oder indirekt über mehr spirituell und teosophisch orientierte Vermittler wie Charles Bragdon und P. D. Uspenskij Einfluss auf Künstler wie Malewitsch, Kandinskij, Picasso, Mondrian und Duchamps aus. ${ }^{25}$ Die hohe Attraktivität des Konzepts bestand vor allem darin, dass die vierte Dimension bei aller wissenschaftlichen Fundierung einen Freiraum für Imagination und Phantasie bot, wie es der Philosoph und Mathematiker Bertrand Russell in seiner Rezension von Hintons The Fourth Dimension 1904 auf den Punkt brachte: »The merit of speculations on the fourth dimension [...] is chiefly that they stimulate the imagination, and free the intellect from shackles of the actual. A complete intellectual liberty would only be attained by a mind which could think as easily of the non-existent as of the existent. « 26

Bei Moore findet sich auch dieser Aspekt des Raums der Imagination, für den er im Briefwechsel mit dem kanadischen Comicautor Dave Sim den Begriff Idea Space prägt:

I would talk in terms of the murders being events not only in the realk material world, but also in the terrain that I term sldea Space, 1 a kind of medium or field or space or dimension in which thoughts occur. I believe this space to be at least in part mutual, rather than discrete, which is to say that I believe that this spacer impinges to some degree upon all consciousness and that it is coaccessible. ${ }^{27}$

In diesem Sinne handelt From Hell nicht von einem realen, faktischen Mordfall, sondern von einem imaginären, fiktiven Kontinuum der Diskurse über den Täter und seine Opfer. Begünstigt durch den >Glücksfall< der nie geklärten Identität Jack the Rippers, gestalten Moore und Campbell in diesem Freiraum ein hochkomplexes Gebilde aus diversen Komponenten des Redens über Jack und seine Untaten, aus denen sie ihre eigene Version zusammenstellen. Dass sie damit selbst Bestandteil dieses Diskurses werden (der z.B. wiederum eine Verfilmung generiert hat), thematisieren sie im Appendix II des Comics, »Dance of the gull catchers «, in dem die endlosen Spekulationen über den >wahren< Ripper schlaglichtartig zusammengefasst und ihre Urheber ironisch als lange Reihe von Tänzern dargestellt werden, die Schmetterlingsnetze nach imaginären Flugobjekten (wie z.B. gulls, Seemöwen) schwingen und untereinander streiten.

\footnotetext{
25 Das Thema ist von der Forschung umfassend aufgearbeitet worden; hervorzuheben sind die Arbeiten von Linda Dalrymple Henderson, v.a.: The Fourth Dimension and Non-Euclidean Geometry in Modern Art. Cambridge rev. ed. 2010. Zum nicht ganz so fruchtbaren Niederschlag des Konzepts in der Literatur vgl. Willard Bohn: »Writing the Fourth Dimension.«In: Comparative Critical Studies 4.1 (2007), S. 121-138, sowie Mark Blacklock: »>On the Eve of the Fourth Dimension.< Utopian Higher Space.« In: Utopian Spaces of Modernism. Literature and Culture, 1885-1945. Hrsg. v. Rosalyn Gregory u. Benjamin Kohlmann. New York 2012, S. 35-51.

${ }^{26}$ Bertrand Russell: »New Books. The Fourth Dimension. By Charles Howard Hinton.« In: Mind 13 (1904), S. 573-574. (Zit. nach Henderson 2011, S. 136.)

27 Sim u. Moore: »Correspondence: From Hell.« A.a.O., S. 315.
} 
Im Comic ist der Idea Space generell nicht nur metaphorisch zu verstehen, sondern nimmt konkrete Formen an. ${ }^{28}$ Seine Einzelbilder repräsentieren dreidimensionale Weltausschnitte, sie werden auf der Seite zweidimensional kombiniert, die Seiten wiederum in der Codexform dreidimensional; damit kommt ein Comic Hintons aus dreidimensionalen geometrischen Formen zusammengesetzten vierdimensionalen Gebilden denkbar nahe, zumal erstere in seinen Schriften zweidimensional dargestellt werden. Der Leser ist in der Lage, zwischen diesen Formen in verschiedene Richtungen (und damit auch Zeiten) Verbindungen herzustellen, über die Seitengrenzen hinweg ebenso wie simultan auf der Einzel- oder Doppelseite. ${ }^{29}$ Moore und Campbell demonstrieren das metaphorisch in der Todesvision Gulls im letzten Kapitel, in der sie ihren Protagonisten sowohl durch den Raum als auch durch die Zeit, in die Vergangenheit ebenso wie in die Zukunft reisen lassen, wobei er intertextuelle wie auch interpiktoriale Verbindungen nicht nur erlebt, sondern teilweise sogar auslöst (vgl. oben Abb. 21). Konkret versuchen sie das mit einer Seite, die einzelne Bilder aus unterschiedlichen, noch nicht dargestellten und zeitlich getrennten Szenen in eine Einheit zusammenführt (II 1, Abb. 22):

\footnotetext{
${ }^{28}$ Mark Bernard und James Bucky Carter sehen den Comic gar als die Kunstform, die die in Kubismus und Futurismus angestrebten Tendenzen zur Vollendung bringt: »What has hitherto been unexplored, however, is how purely sequential art forms utilize aspects of these movements to fulfill the elusive goals and ideals of many of cubism and futurism's most renowned creators via a unique relationship with the space-time continuum. Indeed, no media before or after the comic book, and more specifically, the graphic novel, has fully bridged the fourth dimension as well. Comics and graphic novels, we argue, constitute the 2oth century culmination of the goals of these other pivotal modern and postmodern genres.«(»Alan Moore and the Graphic Novel. Confronting the Fourth Dimension.« In: ImageTexT 1.2 [2005], <http://www.english.ufl.edu/imagetext/ archives/v1_2/carter/> [Zugriff 6.5.2014].)

29 Thierry Groensteen fasst das theoretisch in der Begriffstrias spatio-topia, arthrology und braiding, die unterschiedliche Verbindungsmöglichkeiten zwischen Einzelpanels auf der Seite oder über Seitengrenzen hinweg bezeichnen; vgl. seine Studie The System of Comics. Jackson 2007.
} 

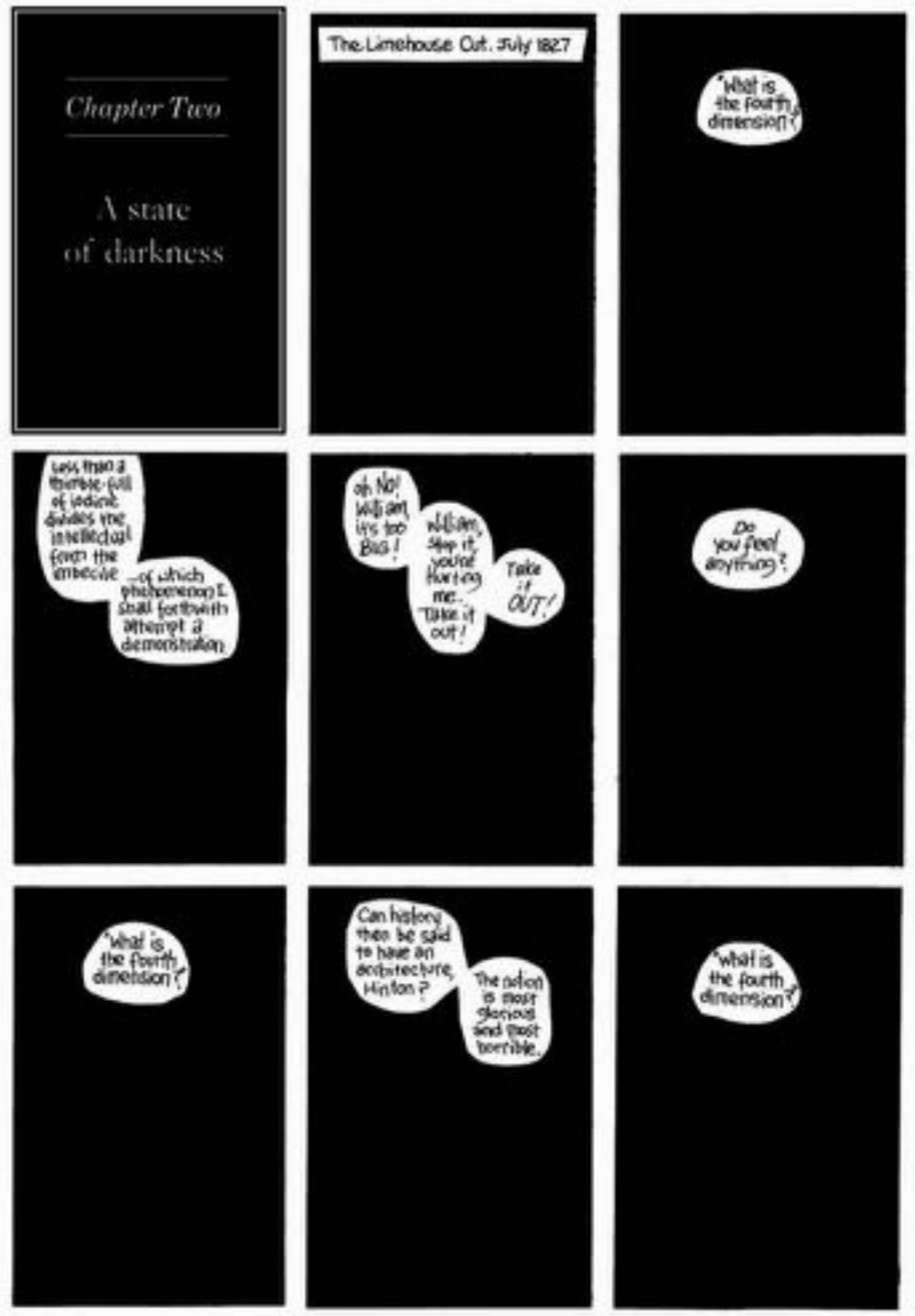

Abb. 22

\section{Literatur}

Acland, Theodor Dyke: William Withey Gull. A Biographical Sketch. London 1896.

Bacon, Francis: De Dignitate et Augmentis Scientiarum Libri IX [1623]. Hg. v. Philipp Mayer. Bd. 1. Nürnberg 1829.

ders.: Über die Würde und den Fortgang der Wissenschaften. Hg. v. Johann Hermann Pfingsten. Pest 1783.

Bernard, Mark u. James Bucky Carter: »Alan Moore and the Graphic Novel. Confronting the Fourth Dimension.« In: ImageTexT 1.2 (2005), <http://www.english.ufl.edu/imagetext/archives/v1_2/ carter/> (Zugriff 6.5.2014).

Bettany, George Thomas: »Gull, William Withey.« In: Dictionary of National Biography. Hrsg. v. Leslie Stephen u. Sidney Lee. Bd. 23. New York u. London 1890, S. 333-334. 
Blacklock, Mark: »>On the Eve of the Fourth Dimension.< Utopian Higher Space.«In: Utopian Spaces of Modernism. Literature and Culture, 1885-1945. Hrsg. v. Rosalyn Gregory u. Benjamin Kohlmann. New York 2012, S. 35-51.

Böhme, Hartmut und Gernot: Das andere der Vernunft. Zur Entwicklung von Rationalitätsstrukturen am Beispiel Kants. Frankfurt/M. 2. Aufl. 1992.

Bohn, Willard: »Writing the Fourth Dimension.« In: Comparative Critical Studies 4.1 (2007), S. 121138.

Campbell, Eddie: »Comics on the Main Street of Culture.«In: ImageTexT 1.2 (2005), <http:// www.english.ufl.edu/imagetext/archives/v1_2/campbell/> (Zugriff am 30.4.2014).

Coppin, Lisa: »Looking Inside Out. The vision as particular gaze in From Hell (Alan Moore \& Eddie Campbell).« In: Image [Q] Narrative 3.1 (2003), <http://www.imageandnarrative.be/inarchive/ uncanny/lisacoppin.htm> (Zugriff am 1.5.2014).

Croly, David Goodman: Glimpses of the Future. Suggestions as to the Drift of Things. New York u. London 1888.

Curtis, Jr., L. Perry: Jack the Ripper and the London Press. New Haven u. London 2001.

Eliade, Mircea: Schamanismus und archaische Ekstasetechnik. Zürich u. Stuttgart 1954.

Groensteen, Thierry: The System of Comics. Jackson 2007.

Haslam, Fiona: From Hogarth to Rowlandson. Medicine in Art in Eighteenth-century Britain. Liverpool 1996.

Henderson, Linda Dalrymple: The Fourth Dimension and Non-Euclidean Geometry in Modern Art. Cambridge rev. ed. 2010.

dies.: »Darstellung und Vorstellung der vierten Dimension in der Kunst und Kultur des 20. Jahrhunderts. « In: Zahlen, Zeichen und Figuren. Mathematische Inspirationen in Kunst und Literatur. Hrsg. v. Andrea Albrecht, Gesa von Essen u. Werner Frick. Berlin u. Boston 2011, S. 128-159.

Hinton, Charles H.: Speculations on the Fourth Dimension. Selected Writings. Hrsg. v. Rudolf v. B. Rucker. New York 1980.

Horkheimer, Max u. Theodor W. Adorno: Dialektik der Aufklärung. Philosophische Fragmente. Frankfurt/M. 1978.

Hutton, Ronald: Shamans. Siberian Spirituality and the Western Imagination. London 2001.

Junge, Torsten u. Dörthe Ohlhoff (Hrsg.): Wahnsinnig genial. Aschaffenburg 2004.

Kaltenbrunner, Karin: »Das Labor als Ort des Schreckens. Zur Repräsentation des Wissenschaftlers im Film.« In: medienimpulse 4 (2011) <http://www.medienimpulse.at/articles/view/368> (Zugriff am 5.5.2014)

Knight, Stephen: Jack the Ripper. The Final Solution. London 1976.

Kreis, Guido: »Eine unmittelbare Erfahrung.«In: Mantik. Profile prognostischen Wissens in Wissenschaft und Kultur. Hrsg. v. Wolfram Hogrebe. Würzburg 2005, S. 37-55.

Merchant, Carolyn: Der Tod der Natur. Ökologie, Frauen und neuzeitliche Naturwissenschaft. München 1994.

Maier, Michael: Atalanta fugiens, hoc est, Emblemata nova de secretis naturae chymica. Faks.-Dr. d. Oppenheimer Orig.-Ausg. von 1618. Kassel 1964.

Maul, Stefan M.: Die Wahrsagekunst im alten Orient. Zeichen des Himmels und der Erde. München 2013.

Moore, Alan, u. Eddie Campbell: From Hell. Being a Melodrama in Sixteen Parts. Paddington 1999.

dies.: The From Hell Companion. Marietta 2013.

Neumann, Ulrich: »Maier, Michael«. In: Neue Deutsche Biographie. Hrsg. von der Historischen Kommission bei der Bayerischen Akademie der Wissenschaften. Bd. 15. Berlin 1987, S. 703-704.

Pearce, J.M.S.: »Sir William Withey Gull (1816-1890).« In: European Neurology 55 (2006), S. 53-56. 
Pegg, Jennifer D.: »Robert James Lees. Visions From Hell?« In: Ripperoo $11<$ http://www.casebook.org/dissertations/ripperoo-lees.html> (Zugriff am 3.5.2014)

Russell, Bertrand: »New Books. The Fourth Dimension. By Charles Howard Hinton.«In: Mind 13 (1904), S. 573-574.

Schnackertz, Hermann Josef: Form und Funktion medialen Erzählens. Narrativität in Bildsequenz und Comicstrip. München 1980.

Sim, Dave u. Alan Moore: »Correspondence: From Hell.« In: Alan Moore. Portrait of an Extraordinary Gentleman. Hrsg. v. smoky man u. Gary Spencer Millidge. Leigh-on-Sea 2003, S. 303-345.

Smolderen, Thierry: Naissances de la bande dessinée. De William Hogarth à Winsor McCay. Paris 2009.

Sonntag, Michael: »Die Zerlegung des Mikrokosmos. Der Körper in der Anatomie des 16. Jahrhunderts. « In: Transfigurationen des Körpers. Spuren der Gewalt in der Geschichte. Hrsg. v. Dietmar Kamper u. Christoph Wulf. Berlin 1989, S. 59-96. 